\title{
Transvenous Rescue of Pacemaker Lead Fragments after Laser Extraction Failure
}

\begin{abstract}
Keywords
Lead extraction; Pacemaker; ICD; Lead fragments; Sna re retrieva system

Abstract

Transvenouslead extractionsmay be very challenging. Experience and a wide variety of extraction tools are essential in successfully managing such procedures. We report the case of a transvenous fragment extraction after an unsuccessful laser lead extraction procedure.
\end{abstract}

\section{Case Report}

A 35 year old male patient was referred after an unsuccessful lead extraction procedure of a dual chamber pacemaker system using a laser sheath. The pacemaker system was initially implanted for sicksinus-syndrome. Lead implant duration was 104 months. The patient was not pacemaker dependent and showed a sufficient spontaneous heart rate without stimulation.

The pacemaker system needed complete removal due to mitral valve endocarditis with positive blood cultures for staphylococcus aureus. The mitral valve showed only slight mitral valve regurgitation and did not require surgical intervention. During the initial laser extraction procedure, severe adhesions led to a lead rupture of the right ventricular lead with the fragment remaining intravascular in the innominate vein and the left subclavian vein. The right atrial lead was abandoned with the tip in the left subclavian vein due to severe fibrotic adhesions (Figure 1a).

A second procedure was planned for extraction of the lead fragments. The intravascular lead fragment was transvenously rescued by using a snare retrieval system (Needle's Eye Snare Retrieval System, Cook Medical Inc., USA) with an access via the right femoral vein. The lead fragment was retracted into the superior vena cava using the snare, where it was possible to securely catch it and safely extract

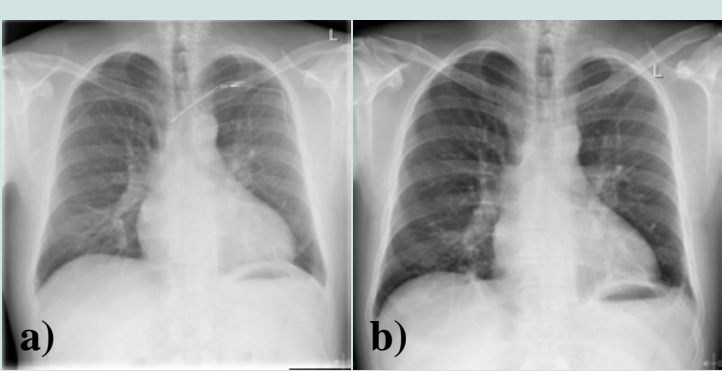

Figure 1: a) Preoperative chest $\mathrm{x}$-ray showing the intravascular lead fragment of the former right ventricular lead and the abandoned fragment of the former right atrial lead. b) Postoperative chest x-ray proving the successful and complete removal of all lead fragments.

\section{Journal of}

\section{Cardiobiology}

Christoph T. Starck ${ }^{1 *}$, Jan Steffel ${ }^{2}$, Felix Schönrath ${ }^{1}$ and Volkmar Falk ${ }^{1}$

${ }^{1}$ Clinic of Cardiac and Vascular Surgery, University Hospital Zurich, Zurich, Switzerland

${ }^{2}$ Clinic of Cardiology, University Hospital Zurich, Zurich, Switzerland

*Address for Correspondence

Dr. med. Christoph T. Starck, Clinic of Cardiac and Vascular Surgery, University Hospital Zurich, Raemistrasse 100, 8091 Zurich, Switzerland, E-mail: christophthomas.starck@usz.ch

Copyright: @ 2014 Starck CT, et al. This is an open access article distributed under the Creative Commons Attribution License, which permits unrestricted use, distribution, and reproduction in any medium, provided the original work is properly cited.

Submission: 24 December, 2013

Accepted: 13 January, 2014

Published: 17 January, 2014

Reviewed \& Approved by: Dr. Li Jianhua

Department of Pediatric Cardiothoracic Surgery, Children's Hospital

Zhejiang University School of Medicine, China

it. The abandoned right atrial lead was intubated with a lead locking device (LLD EZ, Spectranetics, USA) and the fibrotic adhesions were dissected with a mechanical dilator sheath (Evolution, Cook Medical Inc., USA) facilitating complete extraction (Figure 1b). The operation was finished without complications.

Microbiological examination showed that the extracted lead fragments were also positive for staphylococcus aureus, proving lead endocarditis. After an uneventful postoperative course the patient was discharged on the $6^{\text {th }}$ postoperative day. The antibiotic therapy was completed as planned and in compliance with the treatment guidelines of valvular endocarditis.

\section{Discussion}

According to the expert consensus on transvenous lead extractions of the Heart Rhythm Society valvular endocarditis with positive blood cultures are a class I indication for lead extraction, even if evidence of lead involvement is absent [1]. The only predictor of successful treatment of lead endocarditis is complete removal of all foreign material [2].

Pacemaker leads can be extracted either transvenously or surgically in an open procedure. Transvenous lead extractions come with lower operative risk than open surgical removal and can be carried out with high success and low complication rates [3-5]. However transvenous lead extractions may be very challenging due to severe adhesions of implanted leads. In case of lacking experience and restricted extraction tool access success rates dramatically decrease and the risk for major complications increase [5].

In the described case the first procedure failed mainly due to a restricted variety of extraction tools available and used. A lead rupture in situations of severe adhesions may result from a continuous and unchanged action with the same extraction sheath in the same position resulting in damage of the insulation of the lead. This mechanism of action of lead rupture may be less related to the kind of extraction sheath used, but more to the lack of use of different extraction tools, tool sizes and techniques. After lead rupture during 
Citation: Starck CT, Steffel J, Schönrath F, Falk V. Transvenous Rescue of Pacemaker Lead Fragments after Laser Extraction Failure. J Cardiobiol. 2014;2(1): 2.

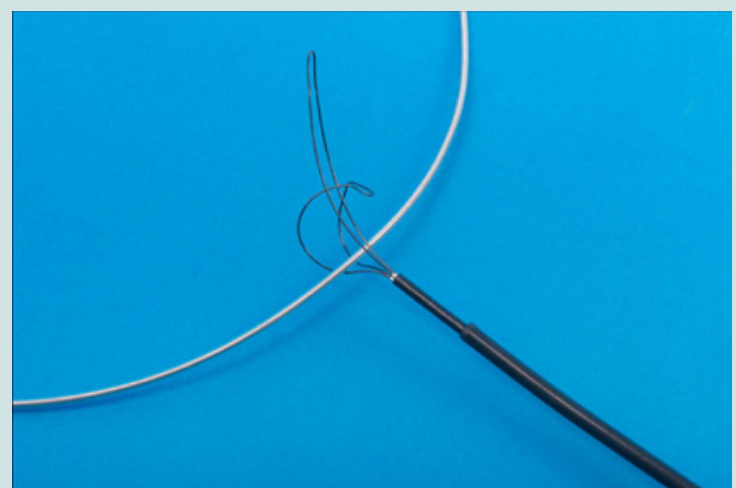

Figure 2: Snare tools (here: Needle's Eye Snare Retrieval System, Cook Medical, USA) facilitate control and successful extraction of completely intravascular leads fragments.

the laser extraction procedure the intravascular remaining fragment could not be retrieved due to a missing snare retrieval system. In this case with an underlying infection a definite abandonment strategy of the lead fragment was no option.

It is important to have a wide variety of extraction tools available, especially tools for a subclavian approach as well as a femoral or internal jugular approach, including snare retrieval systems [6]. In challenging cases success often depends on the liberal use of different extraction tools and techniques. Especially in cases with completely intravascular lead fragments, which are targeted for extraction, snare tools are indispensable to get control of the lead and to facilitate extraction (Figure 2). In such cases either a femoral approach (via the right femoral vein) or an internal jugular approach (via the right internal jugular vein) enables good access to the targeted lead.

Success rates of lead extractions are related to the operator's experience [5]. Therefore lead extractions should be restricted to especially trained physicians and a minimum of 20 lead extractions should be performed annually in order to maintain the required skills [1].

In centers where lead extractions are performed, it is important to have a lead extraction protocol which is strictly followed in order to assure a maximum of patient safety [6]. Extraction procedures should be performed in an operating room environment with the possibility of immediate emergency thoracotomy in case of major complications and such patients need an extensive monitoring during and immediately after the procedure including electrocardiography, invasive blood pressure measurement and transesophageal echocardiography.

\section{Conclusion}

Successful lead extraction procedures depend on sufficient experience of the performing physician, the availability and use of different extraction tools and the existence of and the strict adherence to a standard extraction protocol. When respecting these principles it is possible to achieve high success rates with complete extractions and low complication rates.

\section{References}

1. Wilkoff BL, Love CJ, Byrd CL, Bongiorni MG, Carrillo RG, et al. (2009) Transvenous lead extraction: Heart Rhythm Society expert consensus on facilities, training, indications, and patient management: this document was endorsed by the American Heart Association (AHA). Heart Rhythm 6: 10851104.

2. del Río A, Anguera I, Miró JM, Mont L, Fowler VG Jr, et al. (2003) Surgical treatment of pacemaker and defibrillator lead endocarditis: the impact of electrode lead extraction on outcome. Chest 124: 1451-1459.

3. Maytin M, Epstein LM, Henrikson CA (2010) Lead extraction is preferred for lead revisions and system upgrades: when less is more. Circ Arrhythm Electrophysiol 3: 413-424

4. Kennergren C, Bjurman C, Wiklund R, Gäbel J (2009) A single-centre experience of over one thousand lead extractions. Europace 11: 612-617.

5. Wazni O, Epstein LM, Carrillo RG, Love C, Adler SW, et al. (2010) Lead extraction in the contemporary setting: the LExICon study: an observational retrospective study of consecutive laser lead extractions. J Am Coll Cardiol 55: 579-586.

6. Kratz JM, Toole JM (2010) Pacemaker and internal cardioverter defibrillator lead extraction: a safe and effective surgical approach. Ann Thorac Surg 90: 1411-1417.

\section{Acknowledgements}

Dr. Christoph Starck and Dr. Jan Steffel received workshop honoraria from Cook Medical Europe Ltd. 